\title{
Papillary glioneuronal tumor (PGNT) exhibits a characteristic methylation profile and fusions involving PRKCA
}

Yanghao $\operatorname{Hou}^{1} *$, Jorge Pinheiro ${ }^{2}$, Felix Sahm ${ }^{1,3,4}$, David E. Reuss ${ }^{1,3}$, Daniel Schrimpf ${ }^{1,3}$, Damian Stichel ${ }^{1,3}$, Belén Casalini $^{1,3}$, Christian Koelsche ${ }^{5}$, Philipp Sievers ${ }^{1,3}$, Annika K. Wefers ${ }^{1,3}$, Annekathrin Reinhardt ${ }^{1,3}$, Azadeh Ebrahimi $^{1,3}$, Francisco Fernández-Klett ${ }^{1,3}$, Stefan Pusch ${ }^{1,3}$, Jochen Meier ${ }^{1}$, Leonille Schweizer ${ }^{6,7}$, Werner Paulus ${ }^{8}$, Marco Prinz ${ }^{9,10,11}$, Christian Hartmann ${ }^{12}$, Karl H. Plate ${ }^{13,14}$, Guido Reifenberger ${ }^{15}$, Torsten Pietsch ${ }^{16}$, Pascale Varlet $^{17}$, Mélanie Pagès ${ }^{17}$, Ulrich Schüller ${ }^{18,19,20}$, David Scheie ${ }^{21}$, Karin de Stricker ${ }^{21}$, Stephan Frank ${ }^{22}$, Jürgen Hench $^{22}$, Bianca Pollo ${ }^{23}$, Nirosha Suraweera ${ }^{24}$, Andreas Unterberg ${ }^{25}$, Stefan M. Pfister ${ }^{4,26,27}$, David T.W. Jones ${ }^{4,28}$, , Andrey Korshunov ${ }^{1,3,4}$, Wolfgang Wick ${ }^{3,29}$, David Capper ${ }^{6,7}$, , Ingmar Blümcke ${ }^{30}$, Andreas von Deimling ${ }^{1,3}$ *, Luca Bertero $^{1,31} *$

\section{*These authors contributed equally}

1 Department of Neuropathology, Institute of Pathology, University Hospital Heidelberg, Heidelberg, Germany

2 Department of Pathology, Centro Hospitalar São João, Porto, Portugal

3 Clinical Cooperation Unit Neuropathology, German Consortium for Translational Cancer Research (DKTK), German Cancer Research Center (DKFZ), Heidelberg, Germany

4 Hopp Children's Cancer Center Heidelberg (KiTZ), Heidelberg, Germany

5 Department of General Pathology, Institute of Pathology, University Hospital Heidelberg, Germany

6 Charité - Universitätsmedizin Berlin, corporate member of Freie Universität Berlin, Humboldt-Universität zu Berlin, and Berlin Institute of Health, Department of Neuropathology, Berlin, Germany

7 German Cancer Consortium (DKTK), Partner Site Berlin, German Cancer Research Center (DKFZ), Heidelberg, Germany

8 Institute of Neuropathology, University Hospital Münster, Münster, Germany

9 Institute of Neuropathology, Medical Faculty, University of Freiburg, Germany

10 BIOSS Centre for Biological Signalling Studies, University of Freiburg, Germany

11 CIBSS Centre for Integrative Biological Signalling Studies, University of Freiburg, Germany

12 Department of Neuropathology, Medizinische Hochschule Hannover, Hannover, Germany

13 Department of Neuropathology, University Hospital Frankfurt, Frankfurt, Germany

14 German Cancer Consortium (DKTK), Partner Site Frankfurt/Mainz, German Cancer Research Center(DKFZ), Heidelberg, Germany

15 Department of Neuropathology, University Hospital Düsseldorf, Düsseldorf, Germany

16 Department of Neuropathology, University of Bonn, Bonn, Germany.

17 Department of Neuropathology, Hôpital Sainte-Anne, Université Paris V Descartes, Sorbonne Paris Cité, Paris, France. 
18 Department of Neuropathology, University Hospital Hamburg-Eppendorf, Hamburg, Germany

19 Department of Pediatric Hematology and Oncology, University Medical Center Hamburg Eppendorf, Germany

20 Research Institute Children's Cancer Center Hamburg, Germany

21 Department of Pathology, Rigshospitalet, Copenhagen, Denmark

22 Department of Medical Genetics and Pathology, Division of Neuropathology, University Hospital Basel, Basel, Switzerland

23 Department of Neuropathology, Neurological Institute Besta, Milan, Italy

24 Department of Neuropathology, The National Hospital for Neurology and Neurosurgery, London, United Kingdom

25 Division of Experimental Neurosurgery, Department of Neurosurgery, University Hospital Heidelberg, Heidelberg, Germany

26 Division of Pediatric Neurooncology, German Cancer Consortium (DKTK), German Cancer Research Center (DKFZ), Heidelberg, Germany

27 Department of Pediatric Oncology, Hematology, Immunology and Pulmonology, University Hospital Heidelberg, Heidelberg, Germany

28 Pediatric Glioma Research Group, German Consortium for Translational Cancer Research (DKTK), German Cancer Research Center (DKFZ), Heidelberg, Germany

29 Department of Neurology, University Hospital Heidelberg, Heidelberg, Germany

30 Department of Neuropathology, University Hospital Erlangen, Erlangen, Germany

31 Pathology Unit, Department of Medical Sciences, University of Turin, Turin, Italy 


\begin{abstract}
Papillary glioneuronal tumor (PGNT) is a WHO-defined brain tumor entity posing a major diagnostic challenge. Recently, SLC44A1-PRKCA fusions have been described in PGNT. We subjected 28 brain tumors from different institutions histologically diagnosed as PGNT to molecular analysis and morphological analysis. Array-based methylation analysis revealed that $17 / 28$ tumors exhibited methylation profiles typical for other tumor entities, mostly dysembryoplastic neuroepithelial tumor and hemispheric pilocytic astrocytoma. Conversely, 11/28 tumors exhibited a unique profile thus constituting a distinct methylation class PGNT. By screening the extended Heidelberg cohort containing over 25000 CNS tumors, we identified three additional tumors belonging to this methylation cluster, but originally histologically diagnosed otherwise. RNA sequencing for the detection of SLC44A1-PRKCA fusions could be performed on 19 of the tumors, 10 of them belonging to the methylation class PGNT. In two additional cases, SLC44A1-PRKCA fusions were confirmed by FISH. We detected fusions involving PRKCA in all of the cases of this methylation class with material available for analyses: the canonical SLC44A1-PRKCA fusion was observed in 11/12 tumors, while the remaining case exhibited a NOTCH1PRKCA fusion. Neither of the fusions was found in the tumors belonging to other methylation classes. Our results point towards a high misclassification rate of the morphological diagnosis PGNT and clearly demonstrate the necessity of molecular analyses. PRKCA fusions are highly diagnostic for PGNT and detection by RNA sequencing allows identification of rare fusion partners. Methylation analysis recognizes a unique methylation class PGNT irrespective of the nature of the PRKCA fusion.
\end{abstract}

Key words Papillary glioneuronal tumor; SLC44A1; PRKCA; NOTCH1; DNA methylation; RNA sequencing 


\section{Introduction}

Papillary glioneuronal tumor (PGNT) is a rare neoplasm that was first reported by Komori et al in 1998 [10], and introduced as a separate entity in the 2007 World Health Organization (WHO) classification of tumors of the Central Nervous System (CNS) and assigned to WHO grade I [12]. Histologically, it is characterized by a biphasic differentiation pattern comprised of pseudopapillary structures, composed of flat to cuboidal glial fibrillary acidic protein (GFAP)-positive astrocytes lining hyalinized vessels, and interpapillary collections of synaptophysin-positive neurocytes with occasional ganglion cells $[11,13]$. Outcome is usually favorable with a reported median 5 -year progression free survival higher than $80 \%$.[1]

A $t(9 ; 17)$ (q31; q24) translocation was initially identified as the sole karyotypic anomaly in two PGNTs and the respective SLC44A1 (solute carrier family 44, member 1)-PRKCA (protein kinase C alpha) fusion was detected by Reverse transcription-polymerase chain reaction (RT-PCR) and fluorescence in situ hybridization (FISH) analysis [5]. This fusion was further confirmed by FISH analysis in four PGNT cases, while it was not found in 15 cases of potential histological PGNT mimics [17]. More recently, the fusion was also identified by dual color interphase FISH analysis in two out of three PGNT [16] and a PGNT case report found a FGFR1 N546K mutation [8].

DNA methylation profiling has proven to be a powerful tool for tumor classification and identification of molecular subclasses. Moreover, this approach allows refining and improving the accuracy of current histologybased diagnosis of CNS tumors [7, 18-20]. So far, genome-wide DNA methylation profiles of PGNT have not been established. Therefore, PGNT was not recognized by recently published DNA methylation-based brain tumor classifier [6].

In this study, we set out to subject tumors histologically diagnosed as PGNT to DNA methylation analysis, DNA copy-number analysis and RNA sequencing. We aimed at exploring the molecular landscape of this tumor entity to improve diagnostic accuracy.

\section{Materials and methods}

\section{Tissue samples}

We analyzed 31 tumor samples from the following pathology institutions: Berlin, Münster, Erlangen, Freiburg, Hannover, Coimbra, Moscow, Essen, Basel, Frankfurt, Dusseldorf, Milan, Copenhagen, Hamburg, London, Paris and Heidelberg. Twenty-eight of these 31 tumors had been histologically diagnosed as PGNT. The remaining three tumors initially received a different diagnosis, but turned out to exhibit features of a core group defined as PGNT by molecular findings. Two of the tumors with FISH data have been published previously [17] .

A reference group with diagnoses based on DNA methylation analysis was selected from the files in the Department of Neuropathology, Heidelberg. The reference group included the potential morphological mimics of PGNT represented by the methylation classes (MC) dysembryoplastic neuroepithelial tumor (DNT); MC rosette-forming glioneuronal tumor (RGNT); MC ganglioglioma (GG); MC central neurocytoma (CN); $M C$ diffuse leptomeningeal glioneuronal tumor (DLGNT); MC chordoid glioma of the third ventricle (CG); MC low grade glioma (LGG), MYB/MYBL1; MC pleomorphic xanthoastrocytoma (PXA); MC hemispheric pilocytic astrocytoma (PA HEMI); MC midline pilocytic astrocytoma (PA MID); MC posterior fossa pilocytic astrocytoma (PA PF); MC ependymoma RELA fused (EP RELA) and MC normal hemispheric cortex (NORM HEMI)[6]. Each of the reference methylation groups contained 10 samples.

The sampling of tumors and clinical data collection were performed in accordance with standards approved by the local ethical committees.

\section{Morphological and immunohistochemical examination}

We evaluated Hematoxylin-Eosin (H\&E)-stained slides applying the diagnostic criteria provided by the 2016 WHO Classification of Tumors of the Central Nervous System [13]. The following features characteristic of PGNT 
were reviewed and documented: biphasic differentiation pattern, pseudopapillary component, vessel hyalinization, interpapillary neurocytes, ganglion cells and microcalcifications. Immunohistochemistry was provided by the contributing centers if available.

\section{Extraction of DNA and RNA}

A suitable area with tumor cell content exceeding $\geq 70 \%$ was identified on H\&E slides and macrodissection performed by punch biopsy (pfm medical, Köln, Germany). DNA and RNA were extracted from Formalin-Fixed Paraffin-Embedded (FFPE) tissue using the Maxwell 16 FFPE plus LEV DNA Kit and Maxwell 16 LEV RNA FFPE Kit (Promega, Madison, WI, USA) according to protocols supplied with the kits.

\section{Genome-wide DNA methylation profiling}

All tumor samples were submitted to DNA methylation analysis. The Illumina Infinium Human Methylation EPIC (850K) BeadChip array (Illumina, San Diego, CA, USA) was employed, following the manufacturer's instructions. Copy number profile (CNP) analysis was assessed by R-package "conumee" [21] after additional baseline correction (https://github.com /dstichel/coumee).

\section{RNA sequencing and FISH}

RNA could be obtained from 19 samples and upon reverse transcription was subjected to next generation sequencing on a NextSeq 500 (Illumina, San Diego, CA, USA) as previously described [19]. We used deFuse [15] and Arriba (https://github.com/suhrig/arriba/) methods for the detection of gene fusions. Procedure of FISH analyses for two cases has been described previously [17].

\section{Statistical analysis}

DNA methylation data were processed with the R/Bioconductor package minfi (version 1.20) [3]. For unsupervised hierarchical clustering, we selected the 15000 methylated CpG sites with the highest median absolute deviation. Clustering was performed using Ward's linkage method and Euclidean distance as described [18]. The t-SNE plot was computed by the R package Rtsne from 15000 most variable cpg sites across the dataset, 2000 iterations and a perplexity value of 10. Comparison of nominal variables was performed using Fisher Exact test.

\section{Results}

\section{Distinct methylation profile of PGNT entity}

Using the methylation data of 28 tumors having received the diagnosis of PGNT we performed t-SNE (Fig. 1) and cluster analysis (Suppl. figure 1 (Online Resource 1)) together with 130 reference cases from 13 distinct methylation classes. 17 of 28 tumors diagnosed as PGNT clearly clustered to methylation classes of other tumor entities: eight tumors clustered to reference cases for MC DNT, five with MC PA HEMI and a single case with MC PXA in the TSNE analysis (Fig. 1). For the three tumors, analysis of methylation data did not clearly distinguishable between MC PA HEMI and MC DNT in repeated t-SNE analysis. However, 11/28 tumors diagnosed as PGNT formed a novel separate cluster, which we termed as MC PGNT. The MC PGNT has not been identified in previous analyses of brain tumors.

Subsequently, in a large t-SNE plot containing over 25000 methylation profile of CNS tumors from extended Heidelberg cohort, we screened and identified three additional cases (cases 10, 11 and 12 in Table 1, Table 2 and Suppl. table 1(Online Resource 3)) that clustering to our MC PGNT. 


\section{Copy-number variations in the PGNT methylation group}

Copy-number variations of the 14 cases in the MC PGNT are described in Fig. 2 and Table 1. Six cases showed a flat copy number profile and five cases showed a focal gain in a region on 17q including PRKCA (case 3 is shown as an example in Fig. 2a). Case 7 (Fig. 2b) and case 13 exhibited a narrow focal loss at the PRKCA locus on chromosome 17q. Case 4 (Fig. 2c) harbored partial 9q loss and partial 17q gain including the PRKCA locus. Among the five cases harboring the $17 q$ focal gain, recurrent case 8 (Fig. $2 d$ ) exhibited additional gains and losses.

\section{RNA-sequencing and FISH confirms PRKCA as a defining feature of MC PGNT}

19 tumors were suitable for RNA-sequencing, including 10 out of 14 cases from MC PGNT. Fusions involving $P R K C A$ were detected in all 10 tumors belonging to MC PGNT. Nine tumors of MC PGNT carried the canonical SLC44A1-PRKCA gene fusion and one tumor a novel NOTCH1-PRKCA gene fusion. NOTCH1-PRKCA fusion has not been reported previously. None of the 9 tumors of our series belonging to other methylation classes contained either of the fusions. The results of RNA sequencing of tumors in MC PGNT are described in Table 1. FISH analysis from 2/14 tumors from MC PGNT have been published previously [17] .

Furthermore, in analysis of RNA-sequencing performed in 273 brain tumors, by using Arriba method, we revealed the SLC44A1-PRKCA gene fusion in only one central neurocytoma. In this series we detected also a PRKCA-FAT1 fusion and a PRKCA-FAM91A1 fusion in two tumors belonging to methylation class low grade glioma, MYB/MYBL1 [6] and a PRKCA-PTPRS fusion in one peripheral nerve sheath tumor.

\section{Clinical data and morphological features of methylation class PGNT cases}

The clinical data of the cases are documented in Table 1 and Suppl. table 1(Online Resource 3). No clear sex predilection was found and the median age at diagnosis was $16(n=13$, range: 6 to 54). All cases with available tumor location were supratentorial, with no clear predilection of lobes. Of these, three cases were located in close proximity to the lateral ventricles. Characteristic morphological features of PGNT were documented in Table 2. All of these cases appeared as low grade neuroepithelial tumor appearance with cellularity varying from case to case. Vessel hyalinization was prominent, as well as the presence of interpapillary neurocytes. The characteristic biphasic differentiation pattern of PGNT was diffusely present in 8/14 cases. Ganglion cells were identified focally only in 3 cases on H\&E stained sections. Microcalcifications were present in 5 of 14 cases. Occasional mitotic figures could be found, but no necrosis or microvascular proliferation occurred in any of these cases. Histologically, we present one typical PGNT (Fig. 3 a, b, c), 3 PGNTs with atypical morphology (Fig.3 d, e, f), and 3 PGNT mimics (Fig. 3 g, h, i). 


\section{Discussion}

From a series of 28 tumors diagnosed as PGNT on morphological grounds, we extracted a core set of 11 tumors exhibiting a unique molecular fingerprint thereby defining a distinct tumor entity. This molecular fingerprint relied on both: a highly characteristic methylation profile and invariable detection of a gene fusion involving PRKCA. Based on the methylation profile this group was termed MC PGNT. The remaining 17 tumors belonged to other well-established entities. Comparing further tumors in our research database with this highly characteristic methylation profile we detected three other cases exhibiting features of the MC PGNT. RNA sequencing revealed a SLC44A1-PRKCA fusion in all of these three cases.

\section{Frequency of $P R K C A$ fusions in PGNT and other brain tumors}

PRKCA encodes protein kinase $C$ alpha (PKC $\alpha$ ), which is a family member of calcium and phospholipiddependent serine/threonine kinases involved in tumor formation and progression [14]. PGNT has been recognized as a distinct tumor entity and subsequently included into the WHO Classification of tumors of the central nervous system $[10,11]$. However, the diagnosis on morphological grounds is difficult with a major problem being the separation from dysembryoplastic neuroepithelial tumors [4]. More recently, the SLC44A1PRKCA fusion has been detected in PGNT $[5,16,17]$. The frequency of this fusion in PGNT varies among studies mainly due to the low number of tumors examined. In the first study, 2 of 3 PGNT carried a SLC44A1-PRKCA fusion [5]. Subsequent analyses found this alteration in 4 of 4[17] and in 2 of 3 PGNT [16]. In our series, 9 of 18 morphologically defined PGNT which could be examined by RNA sequencing or FISH exhibited a fusion involving PRKCA approximating frequency of 50\%. However, employing methylation-based diagnoses this association changed dramatically to 12 PRKCA fusions in 12 tumors allotted to MC PGNT. Our observation of PRKCA fusions in $100 \%$ of MC PGNT raises the question whether the lower frequency of this alteration in previous series was caused by morphology-based misinterpretation of some other tumor entities as PGNT.

In our series of 273 brain tumors submitted to RNA sequencing we detected fusions involving PRKCA in only four cases and none of them was recurrent. However, PRKCA mutations have been described to consistently occurring in chordoid glioma [9]. These tumors characteristically carry a PRKCA D463H point mutation. Analysis of chordoid glioma by methylation analysis demonstrates a profile distinct from that of methylation class PGNT.

\section{Re-definition of PGNT}

PRKCA fusions invariably occur in tumors of MC PGNT but very rarely in other entities. Tumors diagnosed on morphological grounds as PGNT but not harboring a fusion involving PRKCA can be readily allotted to other entities by methylation analysis. This constellation clearly demands a redefinition of PGNT. In a lesion exhibiting features of glioneuronal tumors, PGNT should be diagnosed either if tumors based on methylation analysis are sorted to MC PGNT or if a fusion involving the PRKCA gene is detected. The invariable association of the fusion event with the distinct methylation profile is underlined by a p-value smaller than 0.0001 (Fisher Exact test, Suppl. figure 2(Online Resource 2)). DNA methylation analysis appears to identify the same set of tumors, independent of the gene partnering with PRKCA in the fusions and is more adapted to diagnostic workflows, requiring DNA extracts only. We suggest the future designation "Papillary Glioneuronal Tumor, PRKCA-fused". Without appropriate testing the designation "Papillary Glioneuronal Tumor, NOS" may be used.

\section{Copy number alterations in MC PGNT}

Overall, the number of copy number alterations in tumors of MC PGNT is low comparable to many benign tumors of the central nervous system [7]. The canonical SLC44A1-PRKCA fusion results from a translocation $\mathrm{t}(9 ; 17)(q 31 ; q 24)$. Analysis of CNV of tumors belonging to MC PGNT reveals focal abnormalities on the chromosomal arm 17q involving the PRKCA locus in 7 of 14 cases, possibly as a genomic 'scar' of the translocation formation. Typical examples of focal gains are depicted in Figure 2a, $d$. In contrast, a focal CNV of the 17q PRKCA locus together with chromosome 6, 11 and 12 gains was found in only one of 17 tumors not belonging to MC PGNT. Therefore, such alterations cannot be taken as a definite proof for a SLC44A1-PRKCA fusion; however, they can be considered as indirect evidence for such a fusion gene. Comparable copy number 
alterations are seen in pediatric ependymomas carrying the YAP-MAMLD1 fusion and exhibiting focal alterations of the YAP1 locus at 11q22.1-11q21.2 and MAMLD1 locus Xp28.[2]

\section{Conclusion}

We describe the invariable coincidence of a specific methylation profile with the presence of a fusion gene involving PRKCA in PGNT. All tumors histologically diagnosed as PGNT exhibiting neither of these traits could be identified as belonging to other tumor entities by DNA methylation-based classification. We propose to redefine PGNT with presence of either the methylation profile of MC PGNT or the presence of a fusion involving PRKCA of a glioneuronal tumor being stringent criteria for PGNT diagnosis.

\section{Acknowledgements}

We thank V. Zeller, U. Vogel, H. Y. Nguyen, L. Dörner, U. Lass, A. Habel, K. Lindenberg, S. Kocher, R. Quan for their extraordinary technical support and the microarray unit of the DKFZ Genomics and Proteomics Core Facility for Illumina DNA methylation array analysis support. This study was partly supported by the Else Kröner-Fresenius Stiftung (2107_EKES.24) and the Molecular Neuropathology 2.0 study funded by the Deutsche Kinderkrebsstiftung. U.S. is supported by the Fördergemeinschaft Kinderkrebszentrum Hamburg.

\section{Figure Legends}

Figure 1: t-SNE analysis of PGNT and reference tumors.

28 cases with histological diagnosis of PGNT and the three cases retrospectively identified as belonging to MC PGNT are indicated by a black circle each. 14 cases formed a novel distinct methylation group (depicted in grey). 14 cases matched with other reference groups. 3 cases showed no clear distinction between MC DNT and MC PA HEMI in repeated t-SNE analysis, indicated by dual color. PGNT: MC papillary glioneuronal tumor; DNT: MC dysembryoplastic neuroepithelial tumor; RGNT: MC rosette-forming glioneuronal tumor; CG: MC chordoid glioma of the third ventricle; GG: MC ganglioglioma; CN: MC central neurocytoma; DLGNT: MC diffuse leptomeningeal glioneuronal tumor; LGG MYB: MC low grade glioma, MYB/MYBL1; PXA: MC pleomorphic xanthoastrocytoma; PA HEMI: MC hemispheric pilocytic astrocytoma; PA MID: MC midline pilocytic astrocytoma; PA PF: MC posterior fossa pilocytic astrocytoma; EP RELA: MC ependymoma RELA fused; NORM HEMI: normal hemispheric cortex. $\mathrm{X}$ : three cases showed no clear distinction between DNT and PA HEMI in repeated t-SNE analysis.

\section{Figure 2: CNP in MC PGNT}

Gains (a, c and d) and a loss (b) were detected at the PRKCA locus on 17q in Case 3(a), Case 4(c), Case 8(d) and Case $7(\mathbf{b})$. A partial loss at $9 q$ and gain at $17 q$ was revealed in Case 4 (c). The recurrent tumor Case 8(d) exhibited multiple copy number alterations.

Figure 3: Histology and immunohistochemistry of MC PGNT cases (a-f) and mimics (g-i).

In case 2, H\&E (a 200-fold), GFAP (b 200-fold), and synaptophysin staining (c 200-fold) demonstrate the typical morphology. The biphasic differentiation pattern is present with pseudopapillary darker and denser GFAPpositive astrocytic cells with scant cytoplasm in contrast to synaptophysin-positive oligodendroglia-like neurocytes in the interpapillary areas. In case 5, H\&E (d 200-fold) depicts pseudopapillary structures, but no biphasic differentiation pattern is found due to the lack of interpapillary neurocytes. Case 6 H\&E-staining (e 200-fold) shows no clearly evident pseudopapillary structures and no biphasic differentiation pattern. In case 1 , H\&E (f 100 -fold) reveals prominent microcalcifications while typical biphasic differentiation pattern of PGNT are missing. H\&E (g 100-fold) of case 24 exhibits PGNT-like morphology but methylation analysis yielded MC DNT. Perivascular pseudorosettes and a somewhat biphasic differentiation pattern with oligodendroglia-like 
neurocytes mimic PGNT. H\&E (h 200-fold) of case 25 also suggests PGNT but methylation analysis yielded between MC DNT and MC PA HEMI. Notice the similarity to case 6 (e). In case 23 (i 100-fold), synaptophysin positivity in interpapillary areas with low tumor cell content may be misinterpreted as PGNT, but this case belonged to MC DNT.

Table 1: Clinical and molecular features of the 14 cases belonging to methylation class PGNT

N/A: not available. * Fusion detected by RNA-sequencing. ** Fusion detected by FISH.

Table 2: Morphological features of cases belonging to methylation class PGNT

Diffuse presence was documented as ' + ', focal as ' $-/+$ ', absent as ' - '

\section{Supplementary data}

Suppl. figure 1 (Online Resource 1): Unsupervised hierarchical clustering of the overall cohort of cases $(n=31)$ plus reference cases $(n=130)$

PGNT: histological defined papillary glioneuronal tumors; RGNT: MC rosette-forming glioneuronal tumor; DNT: MC dysembryoplastic neuroepithelial tumor; PA MID: MC midline pilocytic astrocytoma; PA PF: MC posterior fossa pilocytic astrocytoma; DLGNT: MC diffuse leptomeningeal glioneuronal tumor; CN: MC central neurocytoma; EP RELA: MC ependymoma RELA fused; PXA: MC pleomorphic xanthoastrocytoma; LGG MYB: MC low grade glioma, MYB/MYBL1; PA HEMI: MC hemispheric pilocytic astrocytoma; NORM HEMI: MC normal hemispheric cortex; GG: MC ganglioglioma; CG: MC chordoid glioma of the third ventricle.

Suppl. figure 2 (Online Resource 2): Fisher Exact test showing a significant association between MC PGNT and presence of fusions involving PRKCA $(p<0.0001)$.

Suppl. table 1 (Online Resource 3): Clinical and molecular characteristics of the whole cohort $(n=31)$.

* identified tumors in large t-SNE plot belonging to MC PGNT, but originally histologically diagnosed otherwise.

\section{Reference}

1 Ahmed AK, Dawood HY, Gerard J, Smith TR (2017) Surgical Resection and Cellular Proliferation Index Predict Prognosis for Patients with Papillary Glioneuronal Tumor: Systematic Review and Pooled Analysis. World neurosurgery 107: 534-541 Doi 10.1016/j.wneu.2017.08.041

2 Andreiuolo F, Varlet P, Tauziede-Espariat A, Junger ST, Dorner E, Dreschmann V, Kuchelmeister K, Waha A, Haberler C, Slavc let al (2018) Childhood supratentorial ependymomas with YAP1-MAMLD1 fusion: an entity with characteristic clinical, radiological, cytogenetic and histopathological features. Brain pathology: Doi 10.1111/bpa.12659

3 Aryee MJ, Jaffe AE, Corrada-Bravo H, Ladd-Acosta C, Feinberg AP, Hansen KD, Irizarry RA (2014) Minfi: a flexible and comprehensive Bioconductor package for the analysis of Infinium DNA methylation microarrays. Bioinformatics 30: 1363-1369 Doi 10.1093/bioinformatics/btu049

4 Blumcke I, Coras R, Wefers AK, Capper D, Aronica E, Becker A, Honavar M, Stone TJ, Jacques TS, Miyata Het al (2018) Challenges in the histopathological classification of ganglioglioma and DNT: microscopic agreement studies and a preliminary genotype-phenotype analysis. Neuropathol Appl Neurobiol: Doi 10.1111/nan.12522 

Bowdino BS, McComb RD (2013) Identification of a novel, recurrent SLC44A1-PRKCA fusion in papillary glioneuronal tumor. Brain pathology 23: 121-128 Doi 10.1111/j.17503639.2012.00612.x

Capper D, Jones DTW, Sill M, Hovestadt V, Schrimpf D, Sturm D, Koelsche C, Sahm F, Chavez L, Reuss DEet al (2018) DNA methylation-based classification of central nervous system tumours. Nature 555: 469-474 Doi 10.1038/nature26000 Capper D, Stichel D, Sahm F, Jones DTW, Schrimpf D, Sill M, Schmid S, Hovestadt V, Reuss DE, Koelsche Cet al (2018) Practical implementation of DNA methylation and copy-number-based CNS tumor diagnostics: the Heidelberg experience. Acta neuropathologica 136: 181-210 Doi 10.1007/s00401-018-1879-y Gessi M, Abdel Moneim Y, Hammes J, Waha A, Pietsch T (2014) FGFR1 N546K mutation in a case of papillary glioneuronal tumor (PGNT). Acta neuropathologica 127: 935-936 Doi 10.1007/s00401-014-1283-1

Goode B, Mondal G, Hyun M, Ruiz DG, Lin YH, Van Ziffle J, Joseph NM, Onodera C, Talevich E, Grenert JPet al (2018) A recurrent kinase domain mutation in PRKCA defines chordoid glioma of the third ventricle. Nature communications 9: 810 Doi 10.1038/s41467-018-02826-8 Komori T, Scheithauer BW, Anthony DC, Rosenblum MK, McLendon RE, Scott RM, Okazaki H, Kobayashi M (1998) Papillary glioneuronal tumor: a new variant of mixed neuronal-glial neoplasm. The American journal of surgical pathology 22: 1171-1183 Louis DN, International Agency for Research on Cancer., World Health Organization. (2007) WHO classification of tumours of the central nervous system. International Agency for Research on Cancer, Lyon

12 Louis DN, Ohgaki H, Wiestler OD, Cavenee WK, Burger PC, Jouvet A, Scheithauer BW, Kleihues P (2007) The 2007 WHO classification of tumours of the central nervous system. Acta neuropathologica 114: 97-109 Doi 10.1007/s00401-007-0243-4 Louis DN, Ohgaki H, Wiestler OD, Cavenee WK, Ellison DW, Figarella-Branger D, Perry A, Reifenberger G, von Deimling A, International Agency for Research on Cancer. (2016) WHO classification of tumours of the central nervous system. International Agency For Research On Cancer, Lyon Martiny-Baron G, Fabbro D (2007) Classical PKC isoforms in cancer. Pharmacological research 55: 477-486 Doi 10.1016/j.phrs.2007.04.001 McPherson A, Hormozdiari F, Zayed A, Giuliany R, Ha G, Sun MG, Griffith M, Heravi Moussavi A, Senz J, Melnyk Net al (2011) deFuse: an algorithm for gene fusion discovery in tumor RNASeq data. PLoS computational biology 7: e1001138 Doi 10.1371/journal.pcbi.1001138 Nagaishi M, Nobusawa S, Matsumura N, Kono F, Ishiuchi S, Abe T, Ebato M, Wang Y, Hyodo A, Yokoo Het al (2016) SLC44A1-PRKCA fusion in papillary and rosette-forming glioneuronal tumors. Journal of clinical neuroscience : official journal of the Neurosurgical Society of Australasia 23: 73-75 Doi 10.1016/j.jocn.2015.04.021

17 Pages M, Lacroix L, Tauziede-Espariat A, Castel D, Daudigeos-Dubus E, Ridola V, Gilles S, Fina F, Andreiuolo F, Polivka Met al (2015) Papillary glioneuronal tumors: histological and molecular characteristics and diagnostic value of SLC44A1-PRKCA fusion. Acta neuropathologica communications 3: 85 Doi 10.1186/s40478-015-0264-5 Reinhardt A, Stichel D, Schrimpf D, Sahm F, Korshunov A, Reuss DE, Koelsche C, Huang K, Wefers AK, Hovestadt Vet al (2018) Anaplastic astrocytoma with piloid features, a novel molecular class of IDH wildtype glioma with recurrent MAPK pathway, CDKN2A/B and ATRX alterations. Acta neuropathologica 136: 273-291 Doi 10.1007/s00401-018-1837-8

19 Sahm F, Schrimpf D, Stichel D, Jones DTW, Hielscher T, Schefzyk S, Okonechnikov K, Koelsche C, Reuss DE, Capper Det al (2017) DNA methylation-based classification and grading system for meningioma: a multicentre, retrospective analysis. The Lancet Oncology 18: 682-694 Doi 10.1016/S1470-2045(17)30155-9

20 Sievers P, Stichel D, Schrimpf D, Sahm F, Koelsche C, Reuss DE, Wefers AK, Reinhardt A, Huang K, Ebrahimi Aet al (2018) FGFR1:TACC1 fusion is a frequent event in molecularly 
defined extraventricular neurocytoma. Acta neuropathologica 136: 293-302 Doi 10.1007/s00401-018-1882-3

21 Sturm D, Witt $H$, Hovestadt V, Khuong-Quang DA, Jones DT, Konermann C, Pfaff E, Tonjes M, Sill M, Bender Set al (2012) Hotspot mutations in H3F3A and IDH1 define distinct epigenetic and biological subgroups of glioblastoma. Cancer cell 22: 425-437 Doi

10.1016/j.ccr.2012.08.024 


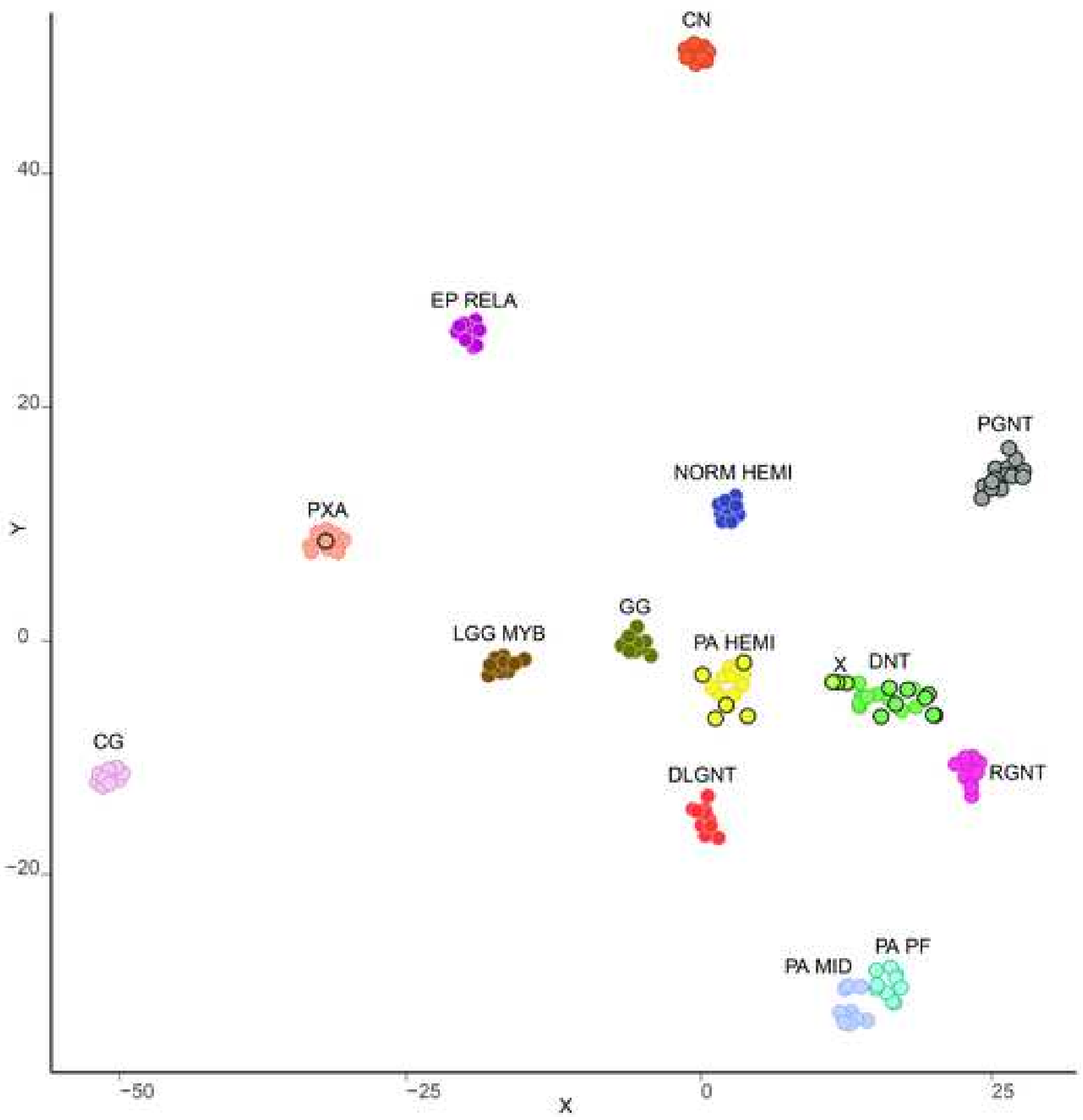



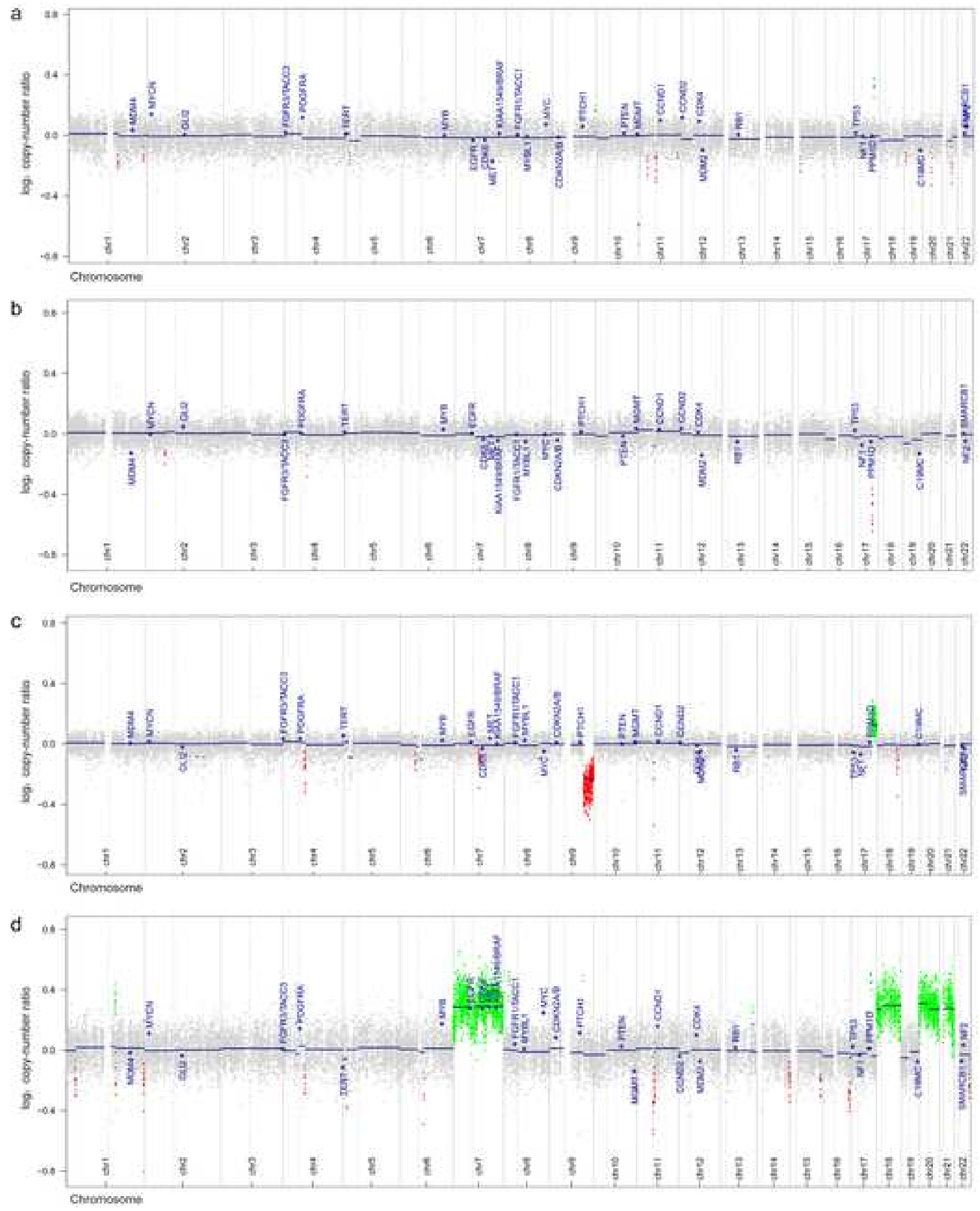


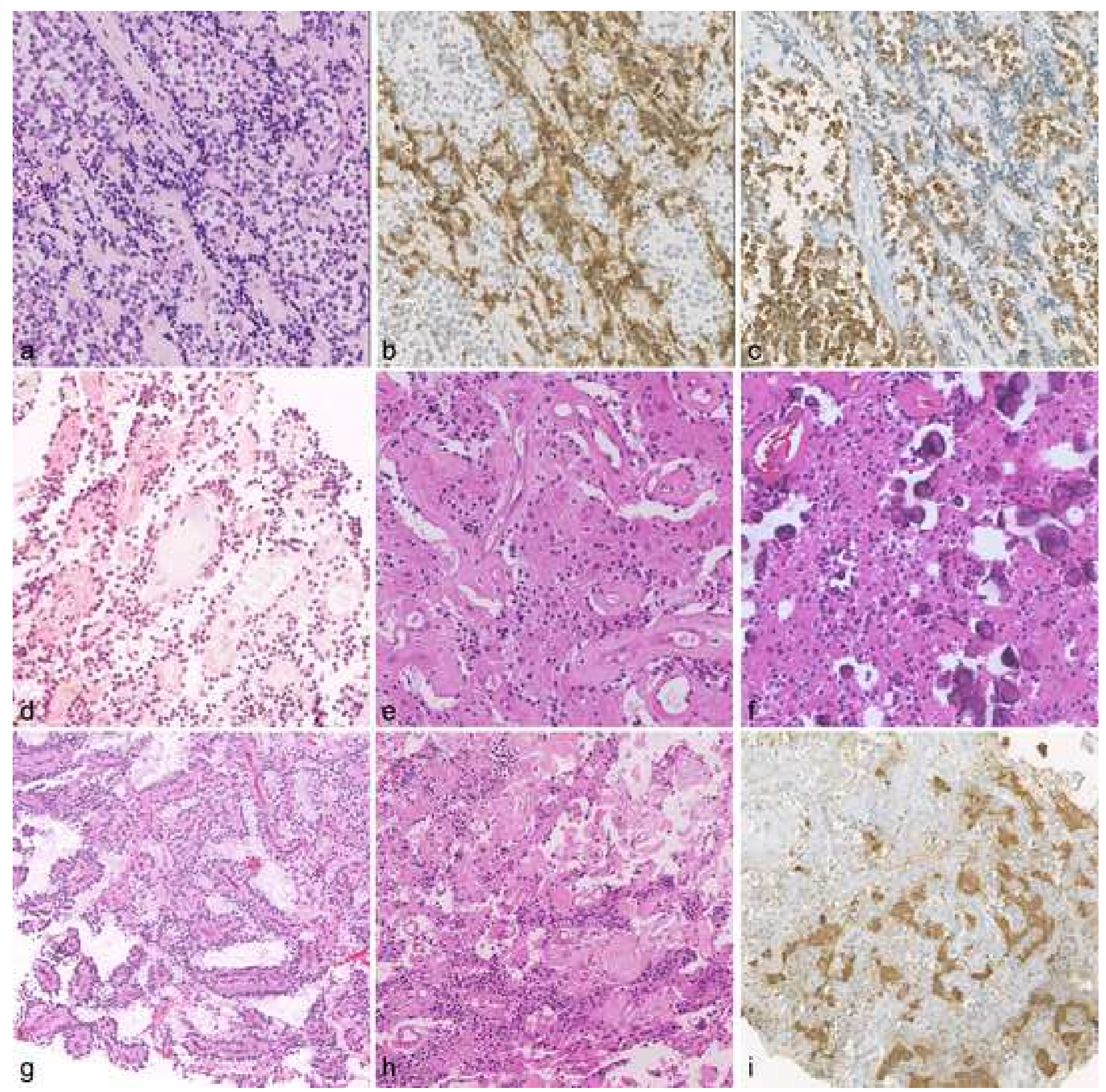




\begin{tabular}{|c|c|c|c|c|c|c|}
\hline $\begin{array}{l}\text { MC } \\
\text { PGNT }\end{array}$ & $\begin{array}{l}\text { Initial } \\
\text { diagnosis }\end{array}$ & $\begin{array}{l}\text { Age } \\
\text { (years) }\end{array}$ & Gender & Location & CNV by $850 \mathrm{~K}$ & Fusion \\
\hline Case 1 & PGNT & 39 & $\mathrm{~F}$ & Left temporal & Flat & $\begin{array}{l}\text { SLC44A1 (exon 15)- } \\
\text { PRKCA (exon 9) * }\end{array}$ \\
\hline Case 2 & PGNT & 17 & $\mathrm{~F}$ & $\begin{array}{l}\text { Left lateral } \\
\text { ventricle }\end{array}$ & Flat & $\begin{array}{l}\text { SLC44A1 (exon 15)- } \\
\text { PRKCA (exon 9) * }\end{array}$ \\
\hline Case 3 & PGNT & 13 & M & $\begin{array}{l}\text { Left mesial } \\
\text { parietal }\end{array}$ & Gain at $17 q$ PRKCA locus & $\begin{array}{l}\text { NOTCH1 (exon 29)- } \\
\text { PRKCA (exon 9) * }\end{array}$ \\
\hline Case 4 & PGNT & 32 & M & $\begin{array}{l}\text { Right parieto- } \\
\text { occipital }\end{array}$ & $\begin{array}{l}\text { Partial loss at } 9 q ; \text { Partial gain } \\
\text { at } 17 q \text { involving } P R K C A \text { locus }\end{array}$ & N/A \\
\hline Case 5 & PGNT & 27 & M & Right temporal & Gain at $17 q$ PRKCA locus & $\mathrm{N} / \mathrm{A}$ \\
\hline Case 6 & PGNT & 15 & $\mathrm{~F}$ & Right frontal & Gain at $17 q$ PRKCA locus & $\begin{array}{l}\text { SLC44A1 (exon 15)- } \\
\text { PRKCA (exon 9)* }\end{array}$ \\
\hline Case 7 & PGNT & 14 & $\mathrm{~F}$ & Supratentorial & Loss at $17 q$ PRKCA locus & $\begin{array}{l}\text { SLC44A1 (exon 15)- } \\
\text { PRKCA (exon 9) * }\end{array}$ \\
\hline Case 8 & $\begin{array}{l}\text { Recurrent } \\
\text { PGNT }\end{array}$ & 54 & M & $\mathrm{N} / \mathrm{A}$ & $\begin{array}{l}\text { Gain 7, 18, 20, } 21 \\
\text { and 17q PRKCA locus }\end{array}$ & $\begin{array}{l}\text { SLC44A1 (exon 15)- } \\
\text { PRKCA (exon 9) * }\end{array}$ \\
\hline Case 9 & PGNT & 16 & M & $\begin{array}{l}\text { Right lateral } \\
\text { ventricle }\end{array}$ & Flat & $\begin{array}{l}\text { SLC44A1 (exon 15)- } \\
\text { PRKCA (exon 9) * }\end{array}$ \\
\hline Case 10 & Ependymoma & 14 & $\mathrm{~F}$ & Intraventricular & Flat & $\begin{array}{l}\text { SLC44A1 (exon 15)- } \\
\text { PRKCA (exon 9) * }\end{array}$ \\
\hline Case 11 & $\begin{array}{l}\text { Central } \\
\text { neurocytoma }\end{array}$ & 16 & $\mathrm{~F}$ & $\begin{array}{l}\text { Left } \\
\text { intraventricular }\end{array}$ & Gain at $17 q$ PRKCA locus & $\begin{array}{l}\text { SLC44A1 (exon 15)- } \\
\text { PRKCA (exon 9) * }\end{array}$ \\
\hline Case 12 & $\begin{array}{l}\text { Glioneuronal } \\
\text { tumor }\end{array}$ & $\mathrm{N} / \mathrm{A}$ & $\mathrm{F}$ & Frontal cortex & Flat & $\begin{array}{l}\text { SLC44A1 (exon 15)- } \\
\text { PRKCA (exon 9) * }\end{array}$ \\
\hline Case 13 & PGNT & 6 & $\mathrm{~F}$ & Left parietal & Loss at $17 q$ PRKCA locus & $\begin{array}{l}\text { SLC44A1-PRKCA } \\
(\text { FISH) ** }\end{array}$ \\
\hline Case 14 & PGNT & 14 & $M$ & Right temporal & Flat & $\begin{array}{l}\text { SLC44A1-PRKCA } \\
\text { (FISH) ** }\end{array}$ \\
\hline
\end{tabular}

N/A: not available. * Fusion detected by RNA-sequencing. ** Fusion detected by FISH. 


\begin{tabular}{|c|c|c|c|c|c|c|}
\hline $\begin{array}{l}\text { MC PGNT } \\
\text { group }\end{array}$ & $\begin{array}{c}\text { Biphasic } \\
\text { differentiation } \\
\text { pattern }\end{array}$ & $\begin{array}{l}\text { Pseudopapillary } \\
\text { structures }\end{array}$ & $\begin{array}{l}\text { Hyalinized } \\
\text { vessels }\end{array}$ & $\begin{array}{l}\text { Interpapillary } \\
\text { neurocytes }\end{array}$ & $\begin{array}{l}\text { Ganglion } \\
\text { cells }\end{array}$ & Microcalcifications \\
\hline Case 1 & - & $-/+$ & + & + & $-/+$ & + \\
\hline Case 2 & $-/+$ & + & + & + & - & - \\
\hline Case 3 & + & + & + & + & $-/+$ & - \\
\hline Case 4 & + & + & + & + & - & - \\
\hline Case 5 & - & + & $-/+$ & - & - & - \\
\hline Case 6 & $-/+$ & $-/+$ & $-/+$ & + & - & + \\
\hline Case 7 & + & + & + & + & $-/+$ & - \\
\hline Case 8 & + & + & + & + & - & - \\
\hline Case 9 & + & + & + & + & - & + \\
\hline Case 10 & + & + & + & + & - & - \\
\hline Case 11 & $-/+$ & - & - & + & - & + \\
\hline Case 12 & $-/+$ & + & $-/+$ & + & - & + \\
\hline Case 13 & + & + & + & + & - & - \\
\hline Case 14 & + & + & + & + & - & - \\
\hline
\end{tabular}

Diffuse presence was documented as ' + ', focal as ' $-/{ }^{\prime}$ ', absent as ' - ' 
Click here to access/download electronic supplementary material ESM_1.tif 
Click here to access/download electronic supplementary material ESM_2.tif 
Suppl. table 1 (Online Resource 3)

Click here to access/download electronic supplementary material ESM_3.docx 